\title{
Feasibility of FDG PET/CT to monitor the response of axillary lymph node metastases to neoadjuvant chemotherapy in breast cancer patients
}

\author{
Marieke E. Straver • Tjeerd S. Aukema • Renato A. Valdes Olmos • \\ Emiel J. T. Rutgers • Kenneth G. A. Gilhuijs • Margaret E. Schot • Wouter V. Vogel • \\ Marie-Jeanne T. F. D. Vrancken Peeters
}

Received: 1 October 2009 /Accepted: 17 November 2009/Published online: 4 February 2010

(C) The Author(s) 2010. This article is published with open access at Springerlink.com

\begin{abstract}
Purpose The aim of this study was to assess the accuracy of ${ }^{18} \mathrm{~F}$-fluorodeoxyglucose (FDG) positron emission tomography (PET)/CT to visualize lymph node metastases before the start of neoadjuvant chemotherapy and to determine how often the visualization is sufficiently prominent to allow monitoring of the axillary response.

Methods Thirty-eight patients with invasive breast cancer of $>3 \mathrm{~cm}$ and/or lymph node metastasis underwent FDG PET/ CT before neoadjuvant chemotherapy. The results of the FDG $\mathrm{PET} / \mathrm{CT}$ were compared with those from ultrasonography with fine-needle aspiration (FNA) cytology or sentinel node biopsy. Patients suitable for response monitoring of the axilla
\end{abstract}

\footnotetext{
M. E. Straver • E. J. T. Rutgers • M.-J. T. F. D. V. Peeters $(\square)$ Department of Surgical Oncology, The Netherlands Cancer Institute-Antoni van Leeuwenhoek Hospital, Plesmanlaan 121 ,

1066 CX Amsterdam, The Netherlands

e-mail: m.vranken@nki.nl

T. S. Aukema $\cdot$ R. A. V. Olmos $\cdot$ W. V. Vogel Department of Nuclear Medicine, The Netherlands Cancer Institute-Antoni van Leeuwenhoek Hospital,

Plesmanlaan 121 ,

1066 CX Amsterdam, The Netherlands

K. G. A. Gilhuijs

Department of Radiology, The Netherlands Cancer

Institute-Antoni van Leeuwenhoek Hospital,

Plesmanlaan 121,

1066 CX Amsterdam, The Netherlands

M. E. Schot

Department of Medical Oncology, The Netherlands Cancer Institute-Antoni van Leeuwenhoek Hospital,

Plesmanlaan 121,

1066 CX Amsterdam, The Netherlands
}

were defined as having either a maximum standardized uptake value $\left(\mathrm{SUV}_{\max }\right) \geq 2.5$ or a tumour to background ratio $\geq 5$ in the most intense lymph node.

Results The sensitivity and specificity of FDG PET/CT in detecting axillary involvement were 97 and $100 \%$, respectively. No difference existed between the $\mathrm{SUV}_{\max }$ of the primary tumour and that from the related most intense lymph node metastasis. Moreover, the mean tumour to background ratio was $90 \%$ higher in the lymph nodes compared to the primary tumour $(p=0.006)$. Ninety-three per cent of the patients had sufficient uptake in the lymph nodes to qualify for subsequent response monitoring of the axilla. A considerable distinction in metabolic activity was observed between the different subtypes of breast cancer. The mean $\mathrm{SUV}_{\max }$ in lymph node metastases of oestrogen receptor (ER)-positive, triple-negative and human epidermal growth factor receptor 2 (HER2)positive tumours was $6.6,11.6$ and 6.6 , respectively.

Conclusion The high accuracy in visualizing lymph node metastases and the sufficiently high $\mathrm{SUV}_{\max }$ and tumour to background ratio at baseline suggest that it is feasible to monitor the axillary response with FDG PET/CT, especially in triple-negative tumours.

Keywords Breast cancer - Axillary lymph node metastasis . FDG-PET/CT · Neoadjuvant chemotherapy

\section{Introduction}

The use of neoadjuvant chemotherapy plays an important role in the treatment of breast cancer. One of the major advantages of neoadjuvant chemotherapy is downstaging of the tumour load. As a result, inoperable advanced tumours may become operable and patients with large operable tumours may be 
offered breast-conserving surgery (BCS) $[1,2]$. Another advantage of neoadjuvant chemotherapy is the possibility to monitor the response of the primary tumour to chemotherapy. Interim evaluation during neoadjuvant chemotherapy creates the opportunity to switch to another regimen in the case of unfavourable response [3]. Furthermore, response monitoring enables and moderates translational research which provides more insight into the behaviour of different tumour subtypes and predictive factors $[4,5]$.

Response monitoring is crucial in the neoadjuvant setting, as it defines the criteria to switch the chemotherapy regimen or to perform early surgery in insufficiently responding tumours at an early time point. After the administration of chemotherapy, response monitoring enables the evaluation of the extent of the residual tumour to select patients for breast-conserving therapy.

The axillary response after neoadjuvant chemotherapy yields prognostic information. Rouzier et al. showed that after neoadjuvant chemotherapy a complete remission of nodal metastases was a strong predictor of disease-free survival (48.7 versus $73.5 \%$ after 5 years) [6]. Early knowledge of the response of axillary lymph node metastases may therefore be helpful to tailor the systemic treatment to individual response. Furthermore, a reliable method to evaluate the axillary tumour response is essential in order to reduce the rate of unnecessary axillary lymph node dissections (ALND), without compromising oncological safety. Patients who achieve a complete remission of their lymph node metastasis could potentially be treated with radiation therapy only. Unfortunately, to date it is not possible to reliably identify these patients. Physical examination and conventional imaging techniques, like MRI and ultrasound, do not have the ability to evaluate the response of axillary lymph node metastasis [7]. Furthermore, the accuracy of the sentinel node procedure after neoadjuvant chemotherapy in patients with proven lymph node metastases remains questionable [8]. At present, the most accurate assessment of the axillary tumour response is an ALND and therefore 'axillaconserving therapy' is not yet among the benefits of neoadjuvant chemotherapy.

Some evidence exists that positron emission tomography (PET)/CT using ${ }^{18} \mathrm{~F}$-fluorodeoxyglucose (FDG) may successfully monitor the response of the primary tumour to neoadjuvant chemotherapy [9-17]. The potential value of FDG PET to assess the response of axillary lymph node metastases to neoadjuvant chemotherapy is, however, currently unknown. The primary aim of this study was to assess the accuracy of FDG PET/CT to visualize lymph node metastases before the start of neoadjuvant chemotherapy. The second aim was to determine how often the visualization is sufficiently prominent to allow monitoring of the axillary response with FDG PET/CT.

\section{Materials and methods}

\section{Patients}

Women who presented with invasive breast cancer larger than $3 \mathrm{~cm}$ in diameter and/or at least one tumour-positive axillary lymph node were scheduled to be treated with neoadjuvant chemotherapy in our institute. Since September 2007 patients were asked to participate in a pilot study to assess the value of FDG PET/CT in the neoadjuvant setting. This study was approved by the Institutional Ethics Committee and informed consent was obtained from all patients.

We analysed 38 patients who had an FDG PET/CT scan before the administration of neoadjuvant chemotherapy. All breast cancers were initially diagnosed by fine-needle aspiration (FNA). Core biopsy was used to determine hormone receptors and human epidermal growth factor receptor 2 (HER2) status and to obtain tumour tissue for expression microarray analysis [5]. The tumour size was assessed with ultrasound, mammography and MRI. Axillary staging was primarily done with ultrasonography and FNA of suspect lymph nodes. In patients with negative lymph nodes (ultrasound and/or FNA cytology negative) a sentinel node biopsy (SNB) procedure was performed prior to neoadjuvant chemotherapy. To assess the presence of distant metastases liver ultrasound, bone scintigraphy and chest X-ray were performed in all patients. Neoadjuvant chemotherapy was followed by BCS or mastectomy. All patients with proven axillary lymph node metastases prior to neoadjuvant chemotherapy underwent an ALND after neoadjuvant chemotherapy. Patients undergoing BCS received radiation to the breast. The indication for locoregional radiation therapy (chest wall and regional nodal basins) was based on the original staging before neoadjuvant chemotherapy. Hormone receptor-positive patients received adjuvant endocrine treatment for at least 5 years and HER2-positive patients received trastuzumab for 1 year.

\section{FDG PET/CT}

The FDG PET/CT scan was performed after conventional imaging of the breast and the axilla. Patients were prepared with a fasting period of $6 \mathrm{~h}$ and $10 \mathrm{mg}$ Valium per os 10 min before FDG administration to avoid brown fat activation. Blood glucose levels were required to be $<10 \mathrm{mmol} / \mathrm{l}$. Patients received $180-240 \mathrm{MBq}$ FDG intravenously. The interval between FDG administrations and scanning was $60 \pm 10 \mathrm{~min}$. A whole-body PET/CT scanner (Gemini TF, Philips, Eindhoven, The Netherlands) was used. FDG PET image acquisition was managed via standardized acquisition procedures. Low-dose CT images 
(40 mAs, 5-mm slices) without oral or intravenous contrast were acquired for anatomical reference and attenuation correction. First, a 2-mm high-resolution PET/CT was performed of the thorax (including breasts and axilla) with the patient in the prone position with hanging breasts and 3 min per bed position. Second, a whole-body PET/CT was performed from the skull base to the groins, with $1.5 \mathrm{~min}$ per bed position and standard image resolution. The administered activity, time of FDG administration and body weight on the day of scanning were recorded for calculation of the tumour maximum standardized uptake value $\left(\mathrm{SUV}_{\max }\right)$.

Image interpretation

All FDG PET/CT scans were read in consensus by three experienced readers. Visual assessments of locations, extent and intensity of FDG uptake patterns were made. The FDG uptake in the primary tumour and lymph node metastases was analysed semi-quantitatively using the $\mathrm{SUV}_{\max }$ and the ratio of the $\mathrm{SUV}_{\text {max }}$ to the mean activity in an adjacent sufficiently large area of normal surrounding tissue (tumour to background ratio, TBR). Patients suitable for response monitoring of the axilla were defined as having either an $\mathrm{SUV}_{\text {max }} \geq 2.5$ or a $\mathrm{TBR} \geq 5$ in the most intense lymph node in the axilla.

\section{Statistical analysis}

The accuracy of FDG PET/CT to visualize lymph node metastasis was evaluated by comparing the results of the FDG PET/CT with the pathological results (tumour-positive cytology or SNB results). The pathologist was blinded to the FDG PET/CT results. The results were classified as true-positive (TP), true-negative (TN), false-positive (FP) and false-negative (FN). The evaluation of the results was based on the sensitivity $[\mathrm{TP} /(\mathrm{TP}+\mathrm{FN})]$, specificity $[\mathrm{TN} /$ $(\mathrm{TN}+\mathrm{FP})]$ and overall accuracy $[(\mathrm{TP}+\mathrm{TN}) /$ all cases $]$.

The differences in $\mathrm{SUV}_{\max }$ and TBRs between the primary tumour and the axillary lymph node metastases were calculated using the paired $t$ test and the Wilcoxon signed rank test. Differences in $\mathrm{SUV}_{\max }$ between the different tumour types were calculated using the one-way analysis of variance (ANOVA) test.

\section{Results}

A total of 38 patients were included in this study. Patient and tumour characteristics are outlined in Table 1. The mean age of the patients was 49 years (range: 30-68). Most patients had T2 $(n=23)$ and ductal tumours $(n=31)$ [18]. Primary tumours were oestrogen receptor (ER)-positive, triple-negative (oestrogen receptor-, progesterone receptorand HER2-negative) and HER2-positive in 19, 17 and 11 patients, respectively. Twenty-nine patients had suspect lymph nodes on ultrasound with tumour-positive FNA cytology. In one patient only a suspect lymph node in the internal mammary chain was palpable (cN2b) and one patient had a positive subclavicular lymph node (cN3), both confirmed by ultrasound-guided FNA cytology.

\section{Positive FDG PET}

FDG PET/CT showed pathological axillary lymph nodes in 30 patients $(86 \%)$. In 27 of these 30 patients lymph node metastasis had also been detected with ultrasound and cytology. In one patient with a negative initial ultrasound examination, FDG PET/CT was positive. Ultrasound was subsequently repeated with FNA cytology, confirming a tumour-positive axillary lymph node (Fig. 1). In two patients with negative ultrasound and a positive FDG $\mathrm{PET} / \mathrm{CT}$ a SNB was done. In one patient the SNB revealed macrometastases in three of four sentinel nodes. In the other patient four lymph nodes were removed during the SNB procedure and they were all tumour-negative. However, follow-up FDG PET/CT images strongly suggested that the suspect lymph nodes were not located in the area of the excised sentinel nodes and had most likely been missed (Fig. 2). We excluded this patient from our accuracy analysis because of uncertainty about the initial lymph node status.

\section{Negative FDG PET}

FDG PET/CT showed negative axillary lymph nodes in eight patients. In one patient the FDG PET/CT was falsenegative because she had a proven axillary lymph node metastasis by ultrasound-guided FNA cytology. Seven patients had a negative ultrasound examination. Four of these patients also had a tumour-negative SNB. In three patients a SNB was not performed. After neoadjuvant chemotherapy, none of these patients had axillary lymph node metastases in the ALND specimen, but we excluded them from our accuracy analysis because of uncertainty about their initial lymph node status.

Feasibility of monitoring the axillary response

The results are summarized in Fig. 3. Excluding the cases outlined above, 34 patients could be evaluated. The sensitivity to visualize axillary lymph node metastasis was $97 \%$ [29/30, 95\% confidence interval (CI): 0.83-0.99], the specificity was $100 \%(4 / 4,95 \% \mathrm{CI}: 0.51-1.0)$ and the overall accuracy was $97 \%$ (33/34). Ninety-three per cent $(29 / 31)$ of the patients had sufficient uptake in the lymph 
Table 1 Patients and tumour characteristics (total $n=38$ )

Number \%

\begin{tabular}{llll}
\hline $\begin{array}{c}\text { Patient age, mean } \\
\text { (range) }\end{array}$ & 49 years (30-68) & & \\
cT classification & cT0 & 1 & 3 \\
& cT1 & 2 & 5 \\
& cT2 & 23 & 61 \\
& cT3 & 10 & 26 \\
& cT4 & 2 & 5 \\
cN classification & cN0 & 9 & 26 \\
& cN1 & 27 & 77 \\
& cN2b & 1 & 3 \\
Histology & cN3 & 1 & \\
& Ductal & 31 & 82 \\
& Lobular & 3 & 8 \\
& Mixed & 1 & 2 \\
& NS & 3 & 8 \\
Receptor status & ER-positive (HER2-negative) & 19 & 51 \\
& Triple-negative (ER-/PR-/ & 7 & 19 \\
& HER2-negative) & & \\
& HER2-positive & 11 & 30 \\
& &
\end{tabular}

$N S$ not specified, $E R$ oestrogen receptor, $P R$ progesterone receptor, $H E R 2$ human epidermal growth factor receptor 2 nodes to qualify for subsequent response monitoring of the axilla, an $\mathrm{SUV}_{\max } \geq 2.5$ or a $\mathrm{TBR} \geq 5$.

Extra-axillary lymph nodes

In 6 of 38 patients (16\%) FDG PET/CT showed suspicious uptake in extra-axillary lymph nodes that were not detected by conventional imaging (Table 2). One patient had two FDG PET-suspect lymph nodes, one intrapectoral and one subclavicular, which were not detected by ultrasound examination. In three patients FDG PET/CT indicated additional lymph node metastases in the internal mammary chain, a region that is not routinely assessed with ultrasound. In one patient FDG PET/CT revealed an unknown subclavicular lymph node metastasis and one patient showed pathological uptake in the contralateral axillary lymph nodes.

Differences between the primary tumour and the lymph node metastasis

The primary tumour could be visualized in 37 of 38 patients. One patient presented with a palpable axillary lymph node and an occult primary tumour that could not be visualized on either FDG PET/CT or MRI. The mean $\mathrm{SUV}_{\text {max }}$ of the primary tumours was 6.8 (range: 1.6-18.8, 95\% CI: 5.1-8.0), with a mean TBR of 11.3 (range: $2.1-$ 36.0, 95\% CI: 8.3-14.3).

Positive lymph nodes (axillary or extra-axillary) were visualized in 31 of the 38 patients. The mean $\mathrm{SUV}_{\max }$ of
Fig. 1 FDG PET scan of a patient without suspect axillary lymph nodes on initial ultrasound examination and a clear tumour-positive lymph node on FDG PET (arrow). Repeated ultrasound examination with cytology guided by FDG PET/ $\mathrm{CT}$ images revealed a tumourpositive axillary lymph node

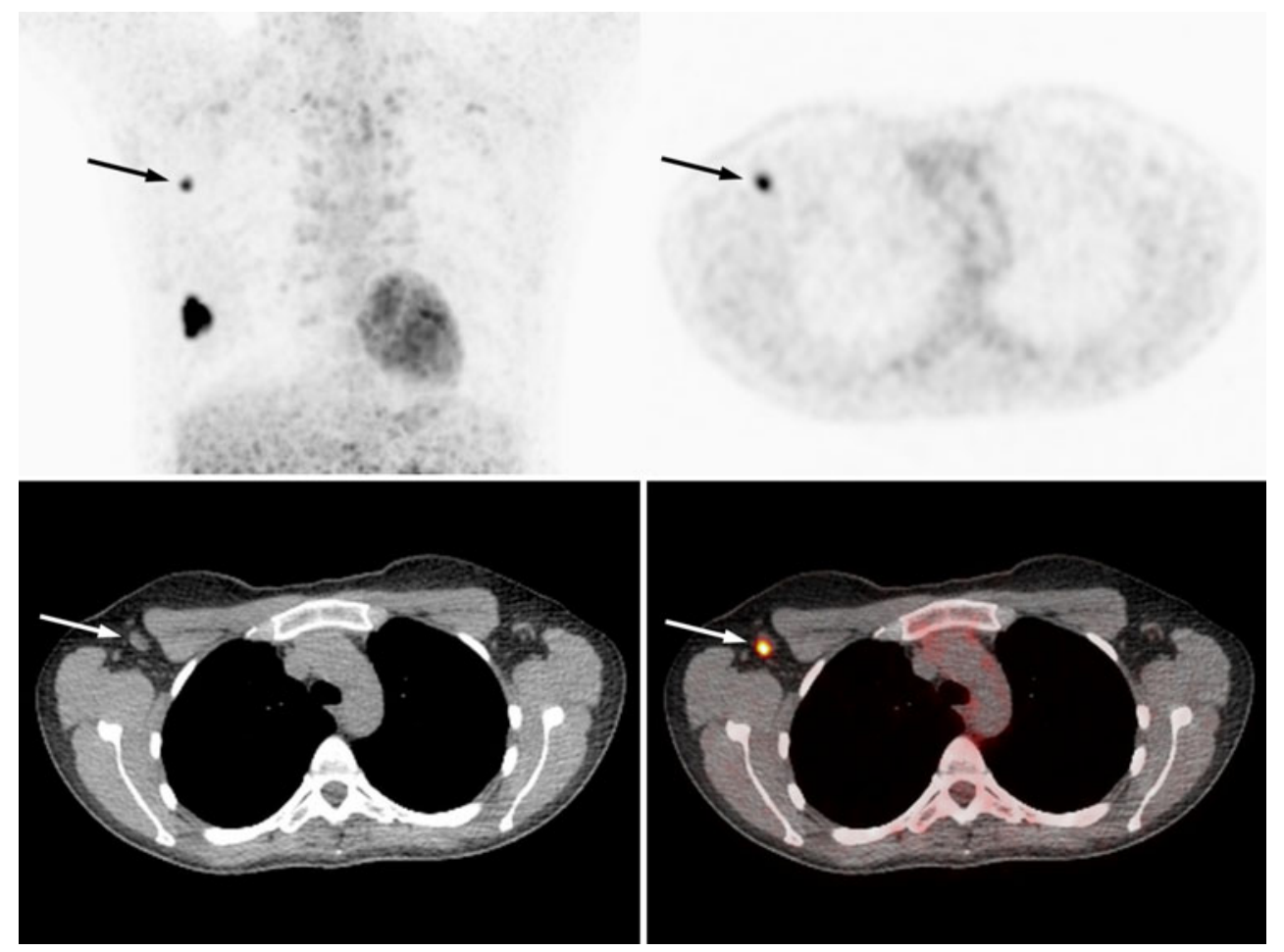



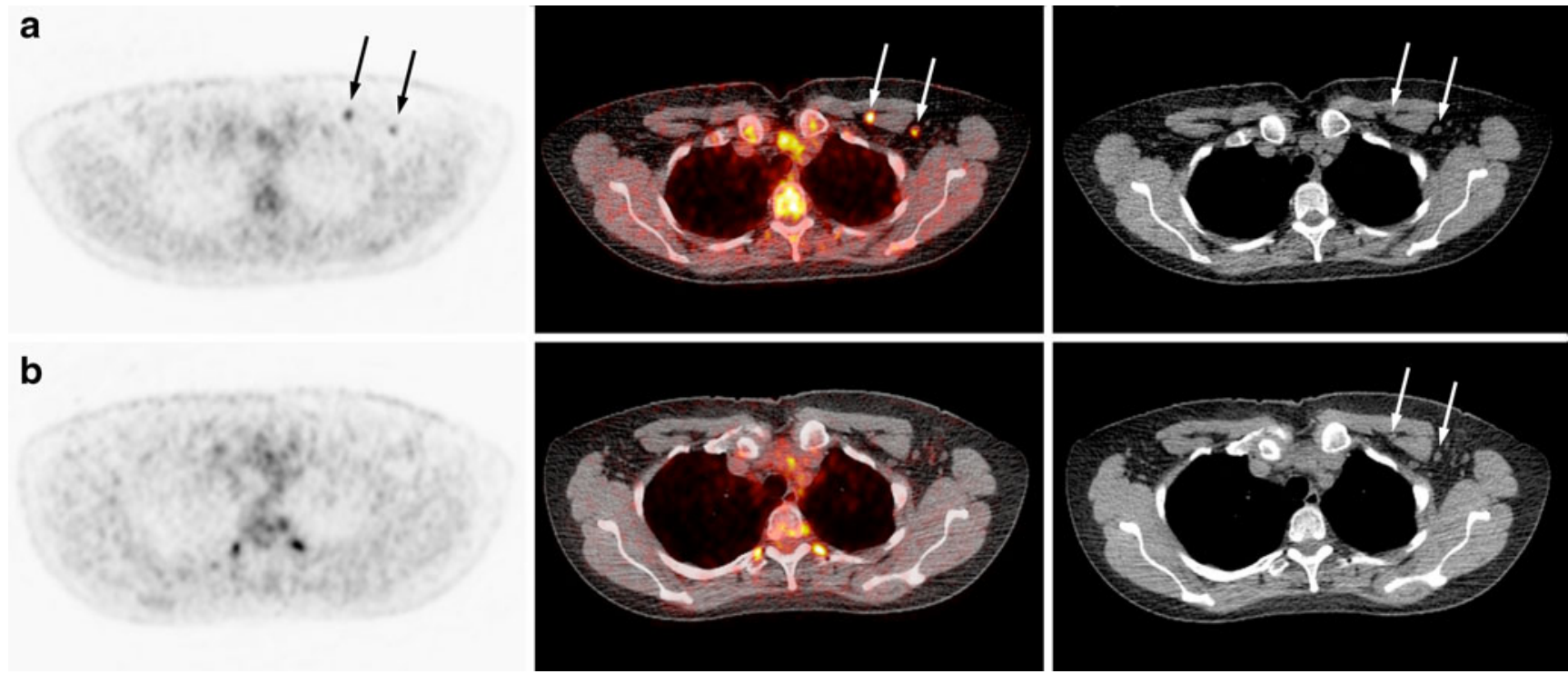

Fig. 2 FDG PET/CT scan of a patient with two FDG PET-positive lymph nodes prior to neoadjuvant chemotherapy. Subsequently, the patient had a negative SNB (a). After the administration of chemotherapy (b) the lymph nodes show complete metabolic response

the most active lymph nodes was 7.7 (range: $2.3-26.2,95 \%$ CI: 5.8-9.6). The mean TBR was 23.7 (range: 4.0-93.0, 95\% CI: 15.2-32.1). Paired evaluation of the $\mathrm{SUV}_{\max }$ of the primary tumour and the related most active lymph node in 30 patients with an active primary tumour and lymph node metastasis showed no difference. Paired evaluation of the TBR showed a significantly higher ratio (2.0) for the lymph nodes $(p=0.006)$, explained by higher background uptake in the breast due to surrounding glandular tissue.

A considerable distinction in the $\mathrm{SUV}_{\max }$ of both the primary tumour and the lymph node metastases was observed between the different subtypes of breast tumours. In ER-positive, triple-negative and HER2-positive tumours the mean $\mathrm{SUV}_{\max }$ was 5.4 (95\% CI: 3.7-7.1), $11.1(95 \%$ CI: $6.0-16.1)$ and $5.9(95 \%$ CI: $3.3-8.6)$, respectively ( $p=$ but are still clearly visible on the low-dose CT and were most likely not removed at SNB. These findings suggest that the patient could have a false-negative SNB procedure or a false-positive initial FDG $\mathrm{PET} / \mathrm{CT}$

0.01). In the lymph node metastases the mean $\mathrm{SUV}_{\max }$ of ER-positive, triple-negative and HER2-positive tumours was 6.6 (95\% CI: 4.7-8.4), 11.6 (95\% CI: 5.0-18.2) and 6.6 (95\% CI: $2.7-10.6)$, respectively $(p=0.07)$.

\section{Discussion}

This report describes the accuracy of FDG PET/CT to visualize lymph node metastases in breast cancer before the start of neoadjuvant chemotherapy. The overall accuracy in this selected patient population was high: $97 \%$. In $93 \%$ of the patients the lymph nodes showed adequate uptake for subsequent response monitoring of the axilla. Accurate visualization of lymph node metastasis with sufficient

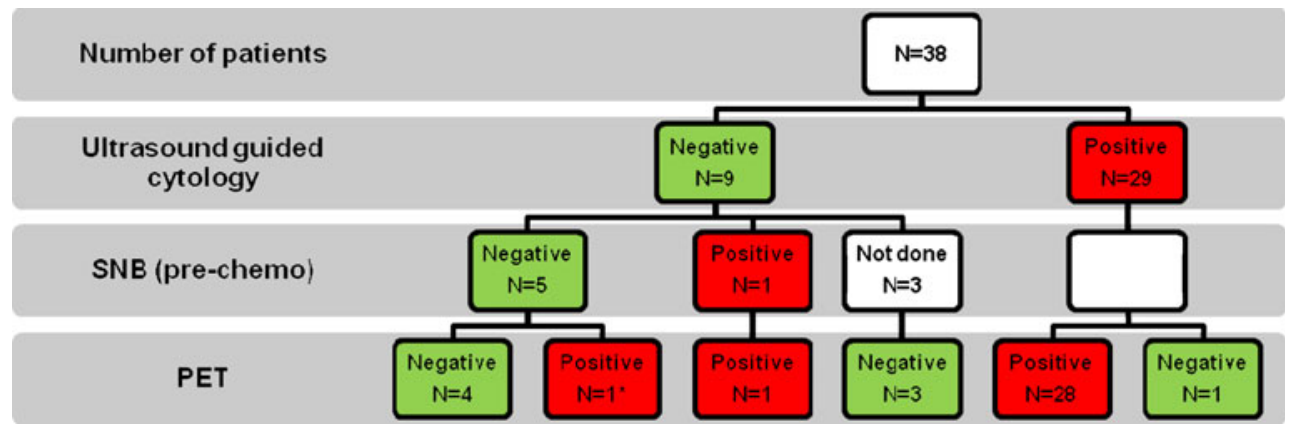

Fig. 3 FDG PET/CT versus conventional examination of the axilla, showing the accuracy of FDG PET/CT to detect lymph node metastases. The sensitivity to visualize axillary lymph node metastasis was $97 \%(29 / 30,95 \%$ CI: $0.83-0.99)$, the specificity was $100 \%(4 / 4$,
95\% CI: 0.51-1.0) and the overall accuracy was $97 \%$ (33/34). *This patient most likely had a false-negative sentinel node and was excluded from the analysis together with the three patients with negative ultrasound-guided cytology and no SNB 
Table 2 Extra-axillary lymph node metastasis in 38 patients

\begin{tabular}{|c|c|c|}
\hline & $\begin{array}{l}\text { Conventional imaging (US/ } \\
\text { FNA positive) }\end{array}$ & $\begin{array}{l}\text { FDG PET/CT } \\
\text { positive }\end{array}$ \\
\hline $\begin{array}{l}\text { Internal } \\
\text { mammary chain }\end{array}$ & 1 & 4 \\
\hline Subclavicular & 1 & 3 \\
\hline Intrapectoral & - & 1 \\
\hline Contralateral & - & 1 \\
\hline Total & $2(5 \%)$ & $9(24 \%)$ \\
\hline
\end{tabular}

US ultrasound, FNA fine-needle aspiration

signal intensity prior to the administration of neoadjuvant chemotherapy is the first requirement to monitor the axillary response with FDG PET/CT imaging. With this study we showed that it is reasonable to use FDG PET/CT to assess the axillary response.

In our series the sensitivity of FDG PET/CT to visualize lymph node metastases prior to neoadjuvant chemotherapy was $97 \%(30 / 31)$. The sensitivity reported by others varies widely (20-94\%) [19]. Studies including patients with more advanced primary tumours, similar to our population, showed a sensitivity of $85 \%$ and higher $[16,20]$. This high sensitivity can be explained by the high a priori likelihood of (voluminous) lymph node metastases. The sensitivity of FDG PET/CT is lower in patients with early breast cancer and a clinically negative axilla. In a prospective study Veronesi et al. compared preoperative FDG PET imaging with SNB and reported a sensitivity of only $37 \%$ [21]. The authors demonstrated that FDG PET was unable to identify micrometastases in the lymph nodes. The low sensitivity and inability to detect micrometastases can be explained by the relatively low spatial resolution of PET imaging, which does not allow detection of the micrometastases found using serial sectioning and immunohistochemistry at pathological assessment of sentinel nodes. Other factors that could influence the sensitivity of FDG PET are intrinsic tumour characteristics like grade and type. There is evidence that sensitivity of FDG PET is higher in certain subgroups. Gil-Rendo et al. showed a sensitivity of $100 \%$ to detect lymph node metastasis in a group of patients with

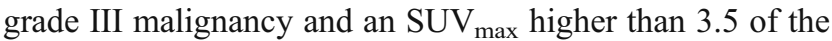
primary tumour [22]. Moreover, Veronesi et al. used FDG PET alone while integrated FDG PET/CT can provide both anatomical and metabolic information, which in general provides a better accuracy [23]. Several studies report the superiority of FDG PET/CT compared with that of PET alone [24-26].

In our series the specificity was $100 \%$. This is confirmed in other studies consistently reporting a high specificity ranging from 85 to $100 \%$ [19]. These findings suggest that an SNB procedure prior to the start of neoadjuvant chemotherapy may not be necessary if FDG PET/CT shows a positive axillary lymph node, at least in the absence of an apparent inflammatory cause of lymph node activation such as non-malignant mastitis. By omitting the SNB procedure prior to neoadjuvant chemotherapy, the positive axillary lymph node will remain in situ, which will create the opportunity to monitor the axillary response in these patients. In addition, the patient is spared an invasive surgical diagnostic procedure.

Fuster et al. reported that FDG PET/CT is a valuable tool to exclude unsuspected extra-axillary lymph node and distant metastases in patients with large breast cancer [27]. They showed that FDG PET/CT led to a change in initial staging in $42 \%$ of patients, although it is critical to note that they did not perform ultrasound assessment combined with cytology of the axillary lymph nodes. In our series, $16 \%$ of the patients had suspect extra-axillary lymph node metastases by FGD PET/CT that were not visualized by conventional imaging (ultrasound examination and SNB). A limitation of our study is that no biopsies were taken of these suspect extra-axillary lymph nodes, but the probability of nodal involvement is high based on the reported specificity of FDG PET/CT.

FDG PET/CT may successfully monitor the response of the primary tumour to neoadjuvant chemotherapy. Quantitative FDG PET scans of primary breast cancers showed a rapid and significant decrease in tumour glucose metabolism after effective treatment was initiated, and the reduction in metabolism antedated the decrease in tumour size $[11,17]$. Several studies investigated the accuracy of FDG PET to monitor the response to neoadjuvant chemotherapy in breast cancer with reported sensitivity and specificity of $84-100 \%$ and $74-94 \%$, respectively [9, 10, 12-16]. These studies are difficult to compare since they use different time points in the course of chemotherapy and different cut-off points in the reduction of the SUV to distinguish responders from non-responders. Overall, it appears that the accuracy of FDG PET to monitor response prediction is high early in the course of therapy. In our study, FDG PET/CT is performed after one cycle of chemotherapy to detect the early response to treatment. However, complete metabolic responders are more likely to occur after a longer course of systemic therapy. Therefore, another scan is performed after the third cycle of chemotherapy. The published studies focused on response monitoring of the primary tumour. No results have been presented yet regarding the value of FDG PET to assess the early response to neoadjuvant chemotherapy of axillary lymph node metastases.

To monitor the early response of axillary lymph node metastases using FDG PET/CT it is crucial to have a sufficiently high baseline $\mathrm{SUV}_{\max }$ or TBR at baseline. A low baseline $\mathrm{SUV}_{\max }$ can underestimate the subsequent 
tumour response [10]. McDermott et al. demonstrated that only tumours with an initial TBR of greater than 5 showed a difference between response categories [13]. Three patients in our study had an $\mathrm{SUV}_{\text {max }}$ lower than 2.5 in the axillary lymph nodes, and three patients had an $\mathrm{SUV}_{\max }$ lower than 2.5 in the primary tumour. The mean TBR was $90 \%$ higher in the axilla than in the primary tumour of the breast, which might facilitate the evaluation of the tumour response in the axillary lymph nodes compared to the primary tumour. The baseline $\mathrm{SUV}_{\max }$ is higher in triplenegative tumours, which will make them more suitable for response evaluation with FDG PET than ER-positive and HER2-positive tumours.

Early axillary response monitoring may have several clinical implications. It seems reasonable that the response of axillary lymph node metastases to the chemotherapy is highly indicative of the efficiency of chemotherapy, particularly because the aim of chemotherapy is to eliminate micrometastatic disease.

The ultimate goal is to adjust the treatment to the early response of the axillary lymph node metastases. Furthermore, knowledge regarding the axillary response might be used to tailor the surgical treatment of the axilla. FDG $\mathrm{PET} / \mathrm{CT}$ might select patients with a favourable axillary response for more axillary-conserving therapies, like axillary radiation therapy. Treatment is still required since PET imaging cannot detect axillary microscopic disease [21].

In conclusion, this study shows that lymph node metastases can be visualized using FDG PET/CT with good sensitivity and specificity. In addition, pathological lymph nodes have a sufficiently high baseline $\mathrm{SUV}_{\max }$ and TBR at baseline to enable response monitoring, especially in triple-negative tumours. Consequently, monitoring the response of axillary lymph nodes with FDG PET/CT during the early course of treatment may be feasible. Future research will focus on establishing a cut-off point in the therapy-induced change of $\mathrm{SUV}_{\max }$ to discriminate nodal responders from non-responders.

Acknowledgement This study was performed within the framework of CTMM, the Center for Translational Molecular Medicine (www. ctmm.nl), project Breast CARE (grant 03O-104).

Open Access This article is distributed under the terms of the Creative Commons Attribution Noncommercial License which permits any noncommercial use, distribution, and reproduction in any medium, provided the original author(s) and source are credited.

\section{References}

1. Mieog JS, van der Hage JA, van de Velde CJ. Neoadjuvant chemotherapy for operable breast cancer. Br J Surg 2007;94 (10):1189-200.
2. Mauri D, Pavlidis N, Ioannidis JP. Neoadjuvant versus adjuvant systemic treatment in breast cancer: a meta-analysis. J Natl Cancer Inst 2005;97(3):188-94.

3. Loo CE, Teertstra HJ, Rodenhuis S, van de Vijver MJ, Hannemann J, Muller SH, et al. Dynamic contrast-enhanced MRI for prediction of breast cancer response to neoadjuvant chemotherapy: initial results. AJR Am J Roentgenol 2008;191 (5):1331-8.

4. Sachelarie I, Grossbard ML, Chadha M, Feldman S, Ghesani M, Blum RH. Primary systemic therapy of breast cancer. Oncologist 2006;11(6):574-89.

5. Hannemann J, Oosterkamp HM, Bosch CA, Velds A, Wessels LF, Loo $\mathrm{C}$, et al. Changes in gene expression associated with response to neoadjuvant chemotherapy in breast cancer. J Clin Oncol 2005;23(15):3331-42.

6. Rouzier R, Extra JM, Klijanienko J, Falcou MC, Asselain B, Vincent-Salomon A, et al. Incidence and prognostic significance of complete axillary downstaging after primary chemotherapy in breast cancer patients with $\mathrm{T} 1$ to $\mathrm{T} 3$ tumors and cytologically proven axillary metastatic lymph nodes. J Clin Oncol 2002;20 (5):1304-10

7. Prati R, Minami CA, Gornbein JA, Debruhl N, Chung D, Chang HR. Accuracy of clinical evaluation of locally advanced breast cancer in patients receiving neoadjuvant chemotherapy. Cancer 2009;115(6):1194-202.

8. Straver ME, Rutgers EJ, Russell NS, Oldenburg HS, Rodenhuis S, Wesseling $\mathrm{J}$, et al. Towards rational axillary treatment in relation to neoadjuvant therapy in breast cancer. Eur J Cancer 2009;45 (13):2284-92.

9. Berriolo-Riedinger A, Touzery C, Riedinger JM, Toubeau M, Coudert B, Arnould L, et al. [(18)F]FDG-PET predicts complete pathological response of breast cancer to neoadjuvant chemotherapy. Eur J Nucl Med Mol Imaging 2007;34(12):1915-24.

10. Duch J, Fuster D, Muñoz M, Fernández PL, Paredes P, Fontanillas $\mathrm{M}$, et al. (18)F-FDG PET/CT for early prediction of response to neoadjuvant chemotherapy in breast cancer. Eur J Nucl Med Mol Imaging 2009;36(10):1551-7.

11. Jansson T, Westlin JE, Ahlström H, Lilja A, Långström B, Bergh J. Positron emission tomography studies in patients with locally advanced and/or metastatic breast cancer: a method for early therapy evaluation? J Clin Oncol 1995;13(6):1470-7.

12. Kim SJ, Kim SK, Lee ES, Ro J, Kang S. Predictive value of [18F] FDG PET for pathological response of breast cancer to neoadjuvant chemotherapy. Ann Oncol 2004;15(9):1352-7.

13. McDermott GM, Welch A, Staff RT, Gilbert FJ, Schweiger L, Semple SI, et al. Monitoring primary breast cancer throughout chemotherapy using FDG-PET. Breast Cancer Res Treat 2007;102 (1) $75-84$

14. Rousseau C, Devillers A, Sagan C, Ferrer L, Bridji B, Campion L, et al. Monitoring of early response to neoadjuvant chemotherapy in stage II and III breast cancer by [18F]fluorodeoxyglucose positron emission tomography. J Clin Oncol 2006;24(34):5366-72.

15. Schelling M, Avril N, Nahrig J, Kuhn W, Römer W, Sattler D, et al. Positron emission tomography using [(18)F]fluorodeoxyglucose for monitoring primary chemotherapy in breast cancer. J Clin Oncol 2000;18(8):1689-95.

16. Smith IC, Welch AE, Hutcheon AW, Miller ID, Payne S, Chilcott F, et al. Positron emission tomography using [(18)F]-fluorodeoxyD-glucose to predict the pathologic response of breast cancer to primary chemotherapy. J Clin Oncol 2000;18(8):1676-88.

17. Wahl RL, Zasadny K, Helvie M, Hutchins GD, Weber B, Cody R. Metabolic monitoring of breast cancer chemohormonotherapy using positron emission tomography: initial evaluation. J Clin Oncol 1993;11(11):2101-11.

18. Sobin LH, Wittekind Ch. International Union Against Cancer, TNM Classification of Malignant Tumours. 6th ed. 2008. 
19. Hodgson NC, Gulenchyn KY. Is there a role for positron emission tomography in breast cancer staging? J Clin Oncol 2008;26 (5):712-20.

20. Greco M, Crippa F, Agresti R, Seregni E, Gerali A, Giovanazzi R, et al. Axillary lymph node staging in breast cancer by 2-fluoro-2deoxy-D-glucose-positron emission tomography: clinical evaluation and alternative management. J Natl Cancer Inst 2001;93 (8):630-5.

21. Veronesi U, De Cicco C, Galimberti VE, Fernandez JR, Rotmensz $\mathrm{N}$, Viale $\mathrm{G}$, et al. A comparative study on the value of FDG-PET and sentinel node biopsy to identify occult axillary metastases. Ann Oncol 2007;18(3):473-8.

22. Gil-Rendo A, Zornoza G, Garcia-Velloso MJ, Regueira FM, Beorlegui C, Cervera M. Fluorodeoxyglucose positron emission tomography with sentinel lymph node biopsy for evaluation of axillary involvement in breast cancer. Br J Surg 2006;93(6):707-12.

23. Antoch G, Saoudi N, Kuehl H, Dahmen G, Mueller SP, Beyer T, et al. Accuracy of whole-body dual-modality fluorine-18-2fluoro-2-deoxy-D-glucose positron emission tomography and computed tomography (FDG-PET/CT) for tumor staging in solid tumors: comparison with CT and PET. J Clin Oncol 2004;22 (21):4357-68.

24. Hany TF, Steinert HC, Goerres GW, Buck A, von Schulthess GK. PET diagnostic accuracy: improvement with in-line PET-CT system: initial results. Radiology 2002;225(2):575-81.

25. Bar-Shalom R, Yefremov N, Guralnik L, Gaitini D, Frenkel A, Kuten A, et al. Clinical performance of PET/CT in evaluation of cancer: additional value for diagnostic imaging and patient management. J Nucl Med 2003;44(8):1200-9.

26. Pelosi E, Messa C, Sironi S, Picchio M, Landoni C, Bettinardi V, et al. Value of integrated PET/CT for lesion localisation in cancer patients: a comparative study. Eur J Nucl Med Mol Imaging 2004;31(7):932-9.

27. Fuster D, Duch J, Paredes P, Velasco M, Muñoz M, Santamaria G, et al. Preoperative staging of large primary breast cancer with [18F]fluorodeoxyglucose positron emission tomography/computed tomography compared with conventional imaging procedures. J Clin Oncol 2008;26(29):4746-51. 